\title{
Gelsolin inhibits the proliferation and invasion of the 786-0 clear cell renal cell carcinoma cell line in vitro
}

\author{
XIAOLING ZHU $^{1 *}$, LIMIN CAI $^{2 *}$, QINGGANG MENG ${ }^{3}$ and XIAOMING JIN ${ }^{4}$ \\ ${ }^{1}$ Department of Dermatology, The First Hospital of Harbin, Harbin, Heilongjiang 150010; ${ }^{2}$ Department of Dermatology, \\ The First Affiliated Hospital of Harbin Medical University, Harbin, Heilongjiang 150089; ${ }^{3}$ Department of Orthopaedic Surgery, \\ The First Hospital of Harbin, Harbin, Heilongjiang 150010; ${ }^{4}$ Department of Pathology, \\ Basic Medical Science College, Harbin Medical University, \\ Harbin, Heilongjiang 150081, P.R. China
}

Received November 12, 2014; Accepted August 17, 2015

DOI: $10.3892 / \mathrm{mmr} .2015 .4313$

\begin{abstract}
The aim of the present study was to investigate the effect of gelsolin (GSN) on the proliferation and invasion of the 786-0 clear cell renal cell carcinoma (ccRCC) cell line in vitro. A GSN overexpression lentiviral vector was constructed and transfected into 786-0 ccRCC cells in vitro. A 3-(4,5-dimethylthiazol-2-yl)-2,5-diphenyltetrazolium bromide (MTT) assay was conducted to detect the effect of GSN on the proliferation and adhesion ability of the 786-0 ccRCC cells, and a Transwell invasion assay was used to determine the effect of GSN on the invasion of 786-0 ccRCC cells. In addition, the expression levels of invasion-associated proteins, matrix metalloproteinase (MMP)2, MMP9 and E-cadherin were analyzed by ELISA and western blotting. The MTT assay demonstrated a significantly lower optical density value for the 786-0/GSN cells compared with that of the 786-0/green fluorescent protein (GFP) and 786-0 cells following 24 - and 48 -h culture $(\mathrm{P}<0.05)$. The mean penetration rate of the 786-0/GSN cells was significantly lower than that of the 786-0/GFP and 786-0 cells $(\mathrm{P}<0.05)$ according to the Transwell invasion assay. The expression levels of MMP2 and MMP9 were significantly decreased in the 786-0/GSN
\end{abstract}

Correspondence to: Dr Qinggang Meng, Department of Orthopaedic Surgery, The First Hospital of Harbin, 151 Diduan Street, Harbin, Heilongjiang 150010, P.R. China

E-mail: 1875199399@qq.com

Dr Xiaoming Jin, Department of Pathology, Basic Medical Science College, Harbin Medical University, 194 Xuefu Road, Harbin, Heilongjiang 150081, P.R. China

E-mail:948174068@qq.com

*Contributed equally

Abbreviations: GFP, green fluorescent protein; GSN, gelsolin; MTT, 3-(4,5-dimethylthiazol-2-yl)-2,5-diphenyltetrazolium bromide

Key words: gelsolin, proliferation, invasion, clear cell renal cell carcinoma cells, when compared with the 786-0/GFP and 786-0 cells following a 48-h transfection, according to ELISA $(\mathrm{P}<0.001)$. Furthermore, in the 786-0/GSN cells, the expression levels of MMP2 and MMP9 were markedly decreased, while the expression of E-cadherin was markedly increased. Thus, the overexpression of GSN may inhibit the proliferation, adhesion ability and invasion of 786-0 ccRCC cells. Additionally, GSN downregulated the expression of MMP2 and MMP9, and upregulated the expression of E-cadherin in the 786-0 ccRCC cells, which may have suppressed the invasion ability of the 786-0 ccRCC cells.

\section{Introduction}

Renal cell carcinoma (RCC) arises from the renal tubular epithelium and accounts for 2-3\% of all adult malignancies in the USA (1). RCC varies widely from region to region, with the highest rates observed in North America and the Czech Republic (2) and it is the tenth most common type of cancer in Europe (3). RCC includes various different histologic subtypes that possess distinct biological behaviors and prognoses (4). Four types of RCC have been clinically characterized, with $85 \%$ of cases of adult RCC being clear cell RCC (ccRCC), while the other $15 \%$ are the papillary, chromophobe and oncocytic types (1). The incidence of ccRCC is steadily increasing by $2.5 \%$ per year, and $>64,700$ new cases and 13,500 mortalities were reported in 2012 (5). Since advanced ccRCC is highly resistant to chemotherapy and cytotoxic therapeutic agents, one-third of patients will experience recurrence following resection of the tumor (6). Furthermore, a quarter of patients present with locally invasive or metastatic RCC (7). Therefore, the factors involved in disease progression and metastasis provide molecular targets to facilitate the development of effective therapeutic strategies.

Gelsolin (GSN) is a widely distributed actin-binding protein, consisting of six domains (G1-G6), which regulates cytoskeletal turnover. GSN has two forms that are encoded by one gene on chromosome 9 in humans: Intracellular (cytoplasmic; cGSN) and extracellular (plasma; pGSN). cGSN is ubiquitously expressed in cells and tissues, and although there are various expression levels during cell differentiation 
and carcinogenesis pGSN is predominantly expressed within muscle cells (8). GSN mediates various cell functions, including cell motility, morphogenesis and actin cytoskeletal remodeling. The most extensively examined roles of GSN are its actin filament severing, capping, uncapping and nucleating activities (9). Furthermore, GSN appears to exert complex roles in tumor biology, with evidence supporting its involvement in tumor suppression and malignant progression. GSN is reported to be downregulated in certain types of tumor, including breast cancer (10) and renal clear cell carcinoma (11), indicating that depletion of GSN promotes oncogenesis. However, to the best of our knowledge, there is no evidence that GSN is involved in the proliferation and invasion of ccRCC. The aim of the current study was to investigate the effect of GSN on the proliferation and invasion of ccRCC by transfection of a GSN overexpression lentiviral vector, pLen0-DCE-RTP-gelsolin, into 786-0 ccRCC cell line cells in vitro.

\section{Materials and methods}

Cell lines and culture. The 786-0 ccRCC cell line and packaging cell line, 293T were purchased from the cell bank of the Chinese Academy of Sciences (Shanghai, China) and cultured in RPMI-1640 (Gibco Life Technologies, Carlsbad, CA, USA) and Dulbecco's modified Eagle's medium (IBCO kit; Gibco Life Technologies) supplemented with $10 \%$ fetal bovine serum (Takara Biotechnology Co., Ltd., Dalian, China) and $1 \%$ penicillin-streptomycin $(100 \mu \mathrm{g} / \mathrm{ml}$; Invitrogen Life Technologies, Beijing, China) for $48 \mathrm{~h}$ at $37^{\circ} \mathrm{C}$ under an atmosphere of $5 \% \mathrm{CO}_{2}$.

Construction of the plenO-DCE-RTP-gelsolin vector and lentivirus packaging. A specific primer was designed using Primer Premier 5.0 software (Shanghai Shenggong Biology Engineering Technology Service, Ltd., Shanghai, China) according to the nucleotide sequences of the human GSN gene, as reported in Genebank (http://www.ncbi.nlm.nih.gov/genbank/; reference sequence: NM_000177.4). The primer sequence for GSN was as follows: Forward, 5'-GGAATTCATGGCTCCGCACCGCCCC-3'; and reverse, 5'-CGGGATCCTCAGGCAGCCAGCTCAG-3'. The coding DNA sequence region of the GSN gene was amplified using a Madison polymerase chain reaction (PCR) kit (Promega Corporation, Madison, WI, USA) according to the manufacturer's instructions. The target DNA gene fragment was subcloned into the pLen0-DCE-RTP lentiviral vector (Baili Biotechnology Co., Ltd., Shanghai, China) to construct a GSN overexpression lentiviral vector (pLen0-DCE-RTP-gelsolin). The GSN was identified by PCR and DNA sequencing. PCR amplification was performed using the following reaction mixture: $1 \mathrm{X}$ PCR reaction buffer $(50 \mathrm{mM} \mathrm{KCl}$ and $20 \mathrm{mM}$ Tris- $\mathrm{HCl}, \mathrm{pH}$ 8.4; Promega Corporation), primers (2.5 $\mu \mathrm{M}$ each), $3 \mathrm{mM} \mathrm{MgCl}_{2}, 0.5 \mathrm{mM}$ dNTPs, $0.4 \mu \mathrm{g}$ template DNA, 1X Q solution and 5 Unit Taq polymerase (Takara Biotechnology Co., Ltd.). The final reaction volume was $50 \mu \mathrm{l}$. The amplification was performed under the following conditions: $94^{\circ} \mathrm{C}$ for $2 \mathrm{~min}, 98^{\circ} \mathrm{C}$ for $10 \mathrm{sec}, 55^{\circ} \mathrm{C}$ for $30 \mathrm{sec}, 68^{\circ} \mathrm{C}$ for $2.5 \mathrm{~min}$ and 30 cycles at $68^{\circ} \mathrm{C}$ for $5 \mathrm{~min}$ using a thermocycler (SimpliAmp ${ }^{\mathrm{TM}}$; Promega Corporation). The PCR products were separated on a $2 \%$ agarose gel (Lonza
Group Ltd., Basel, Switzerland) using 25 and 50 bp molecular weight DNA markers (DL2000TM) and stained with ethidium bromide (Lonza Group Ltd.). The separated gene fragments were analyzed using a iCycler $\mathrm{iQ}^{\mathrm{TM}}$ Real Time PCR Detection and Image Collection system (Lonza Group Ltd.). The images were scanned using a Bio-Rad GelDoc $1000 \mathrm{Gel}$ Documentation system (Bio-Rad Laboratories, Inc., Hercules, CA, USA). The PCR products were purified and sequenced by Gene Co., Ltd. (Beijing, China).

Exponential 293T cells were seeded in $10-\mathrm{cm}$ cell culture dishes $\left(2-2.5 \times 10^{6}\right.$ cells/dish). The lentiviral vector packaging system (volume, 1,800 $\mu \mathrm{l}$ ) was added to the cells at a density of $60-70 \%$. After the supernatant was collected by centrifugation at $30 \mathrm{x} \mathrm{g}$ for $15 \mathrm{~min}$ at $4^{\circ} \mathrm{C}$, the high-concentration lentiviral concentrate was used to infect the $293 \mathrm{~T}$ cells. The ratio of positive cells $\left(2 \times 10^{8} \mathrm{TU} / \mathrm{ml}\right)$ was detected by flow cytometry (FACS Calibur; BD Biosciences, San Jose, CA, USA) and the virus titer was detected using a double dilution assay.

Lentiviral transfection of the 786-0 ccRCC cells. Exponential $786-0$ cells were seeded in 24 -well culture plates $\left(3-5 \times 10^{4}\right.$ cells/well). The viral supernatant with plen0-DCE-RTP-gelsolin and green fluorescent protein (GFP) was added into the cells at a density of $70-80 \%$. After $72 \mathrm{~h}$, the transfection ratio was determined under a fluorescence microscope. The cells with a transfection ratio of $>80 \%$ served as the target cells and were identified by western blot analysis.

There were three experimental groups, including the GSN overexpression group (786-0/GSN), the empty vector group (786-0/GFP) and a control group (786-0). All experiments were performed in triplicate.

MTT assay. The three experimental groups of cells were seeded in 96-well plates at $200 \mu \mathrm{l} / \mathrm{well}\left(1 \times 10^{5}\right.$ cells $\left./ \mathrm{ml}\right)$. Following culturing for $0,12,24,36,48,60$ or 72 h, $20 \mu 1$ MTT $(5 \mathrm{mg} / \mathrm{ml})$ was added to the well. The optical density (OD) value of the cells was determined at a wavelength of 490 nm (GE Healthcare, Uppsala, Sweden).

Cell adhesion assay. Matrigel (Sigma-Aldrich, St. Louis, MO, USA) was added into 96-well plates (50 $\mu \mathrm{g} / \mathrm{well})$. The three groups of cells were resuspended in $0.1 \%$ bovine serum albumin (BSA) RPMI-1640 serum-free medium (Gibco Life Technologies) and added in to the wells with the Matrigel ( $2 \times 10^{4}$ cells/well). The $0.1 \%$ BSA-1640 serum-free medium served as the blank group. There were three repeated wells for each group. Following removal of the non-adherent cells by rinsing with RPMI-1640, the OD value of the cells in each well was determined at a wavelength of $570 \mathrm{~nm}$ using a Thermo Scientific Multiskan ${ }^{\circledR}$ Spectrum spectrophotometer (Bio-Tek Instruments, Inc., Winooski, VT, USA).

Transwell invasion assay. Transwell filters (Corning Incorporated, Corning, NY, USA) were coated with $3.9 \mu \mathrm{g} / \mu \mathrm{l}$ Matrigel (60-80 $\mu \mathrm{l})$. The three groups of cells were resuspended in $100 \mu \mathrm{l}$ serum-free RPMI-1640 medium and added into the upper compartment of the chambers. The cells migrating from the Matrigel into the pores of the inserted filter were fixed with $100 \%$ methanol (Sigma-Aldrich), and stained with hematoxylin (Sigma-Aldrich). The positive-stained cells were 


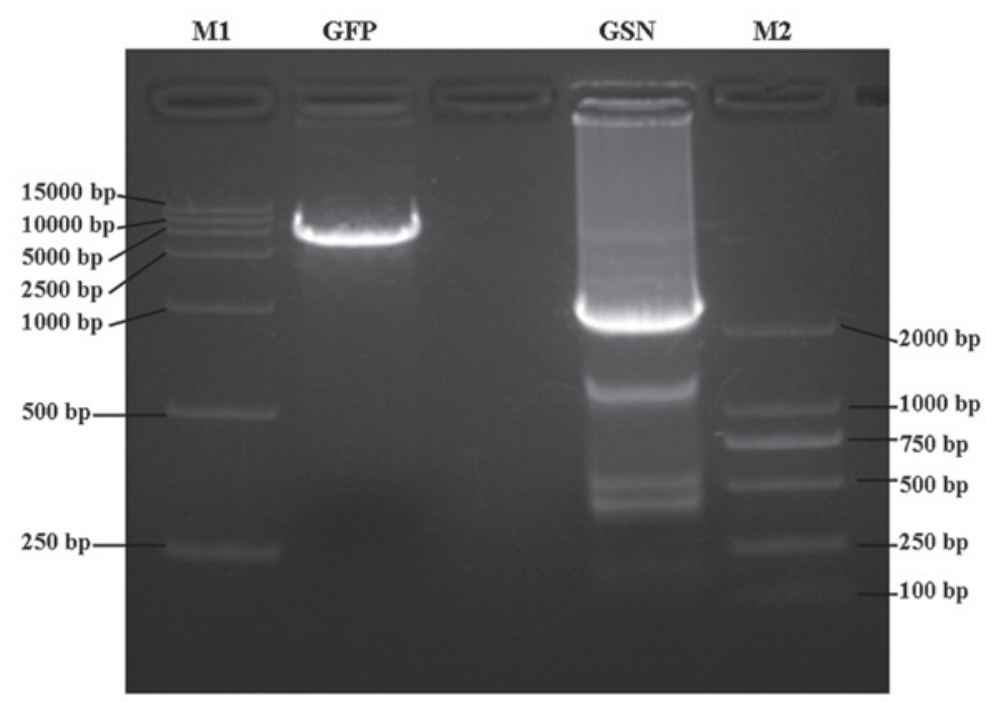

Figure 1. PCR products of the plen0-DCE-RTP-gelsolin vector as analyzed by $1 \%$ agarose gel electrophoresis. The plen0-DCE-RTP-gelsolin vector was constructed and identified by PCR. A single fragment was visualized as a band of $\sim 15 \mathrm{kDa}$. M, maker; GFP, green fluorescent protein; GSN, gelsolin; PCR, polymerase chain reaction.
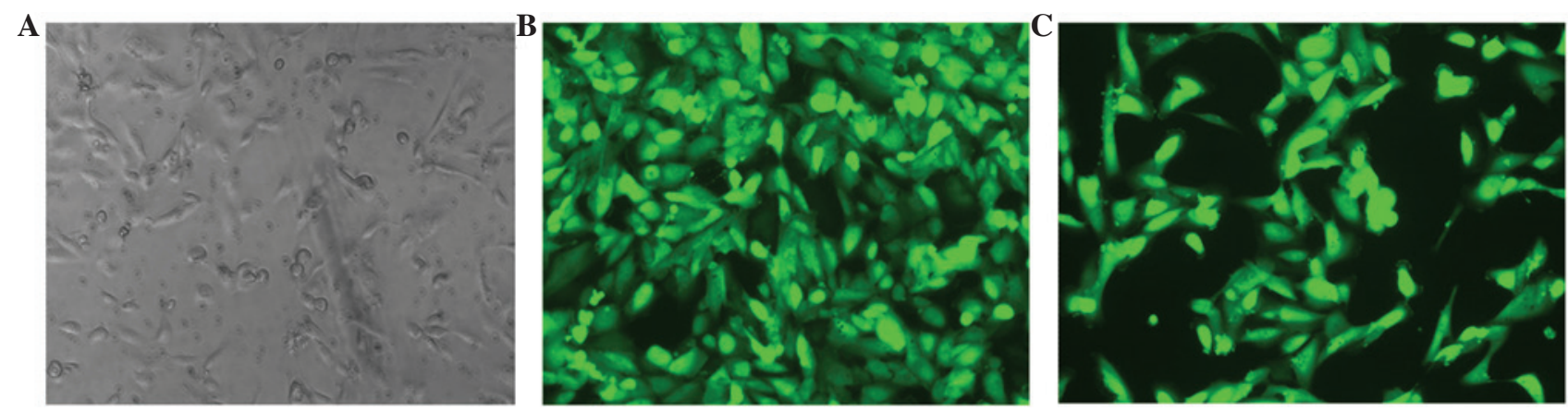

Figure 2. Transfection ratio of the pLen0-DCE-RTP-gelsolin vector in 786-0 clear cell renal cell carcinoma cells observed under a fluorescence microscope (magnification, x200). Green fluorescence in the transfected 786-0 cells indicated a successful transfection. (A) 786-0, non-transfected 786-0 cells; (B) 786-0/GFP, 786-0 cells transfected with GFP; (C) 786-0/GSN, 786-0 cells transfected with the pLen0-DCE-RTP-gelsolin vector. GFP, green fluorescent protein; GSN, gelsolin.

counted under three randomly selected visual fields at $\mathrm{x} 400$ magnification with a fluorescence microscope (SMZ1000; Nikon Corporation, Tokyo, Japan).

ELISA. Three groups of cells were seeded in 96-well plates at $200 \mu \mathrm{l} / \mathrm{well}\left(1 \times 10^{5} \mathrm{cells} / \mathrm{ml}\right)$ and the supernatant was extracted by centrifugation at $30 \mathrm{x} \mathrm{g}$ for $15 \mathrm{~min}$ at $4^{\circ} \mathrm{C}$. The expression levels of matrix metalloproteinase (MMP)2 and MMP9 were determined using ELISA kits (Human MMP-2/Human MMP-9 DuoSet ELISA; R\&D Systems Europe, Ltd., Lille, France) according to the manufacturer's instructions.

Western blot analysis. Total protein was extracted from each of thee three groups of cells using lysis buffer containing $50 \mathrm{mM}$ Tris- $\mathrm{HCl}$ (pH 7.4), $150 \mathrm{mM} \mathrm{NaCl}, 1 \%$ Triton X-100, $0.1 \%$ SDS, and $1 \mathrm{mM}$ EDTA, supplemented with protease inhibitor cocktail (Roche Diagnostics, Basel, Switzerland). Protein $(30 \mu \mathrm{g})$ was subjected to SDS-PAGE and transferred to nitrocellulose membranes (Sigma-Aldrich). Protein samples $(30 \mu \mathrm{g})$ were separated by $10 \%$ SDS-PAGE (BioRad Laboratories, Inc.) and transferred onto nitrocellulose

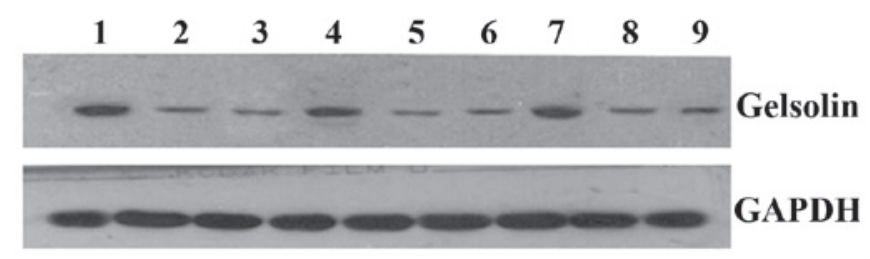

Figure 3. Expression of GSN was examined by western blot analysis. There were three specimens in each group: Lanes 1, 4 and 7, 786-0/GSN; lanes 2, 5 and 8, 786-0/GFP; lanes 3, 6 and 9, 786-0. The expression of GSN was markedly increased in the 786-0/GSN cells compared with the 786-0/GFP and 786-0 cells. GSN, gelsolin; GFP, green fluorescent protein.

membranes (Sigma-Aldrich) for $2 \mathrm{~h}$ at $4^{\circ} \mathrm{C}$ at $200 \mathrm{~mA}$. The membranes were then blocked for $1 \mathrm{~h}$ at room temperature with $5 \%$ non-fat milk. Following electrophoresis of the membranes, the proteins were incubated with primary antibodies against monoclonal mouse anti-human MMP2 (cat. no. MAB-0244), monoclonal mouse anti-human MMP9 (cat. no. MAB-0245) (Cell Signaling Technology, Inc., Danvers, MA, USA), monoclonal mouse anti-human E-cadherin (clone: NCH-38; Dako, Carpinteria, CA, USA) and monoclonal mouse anti-human 

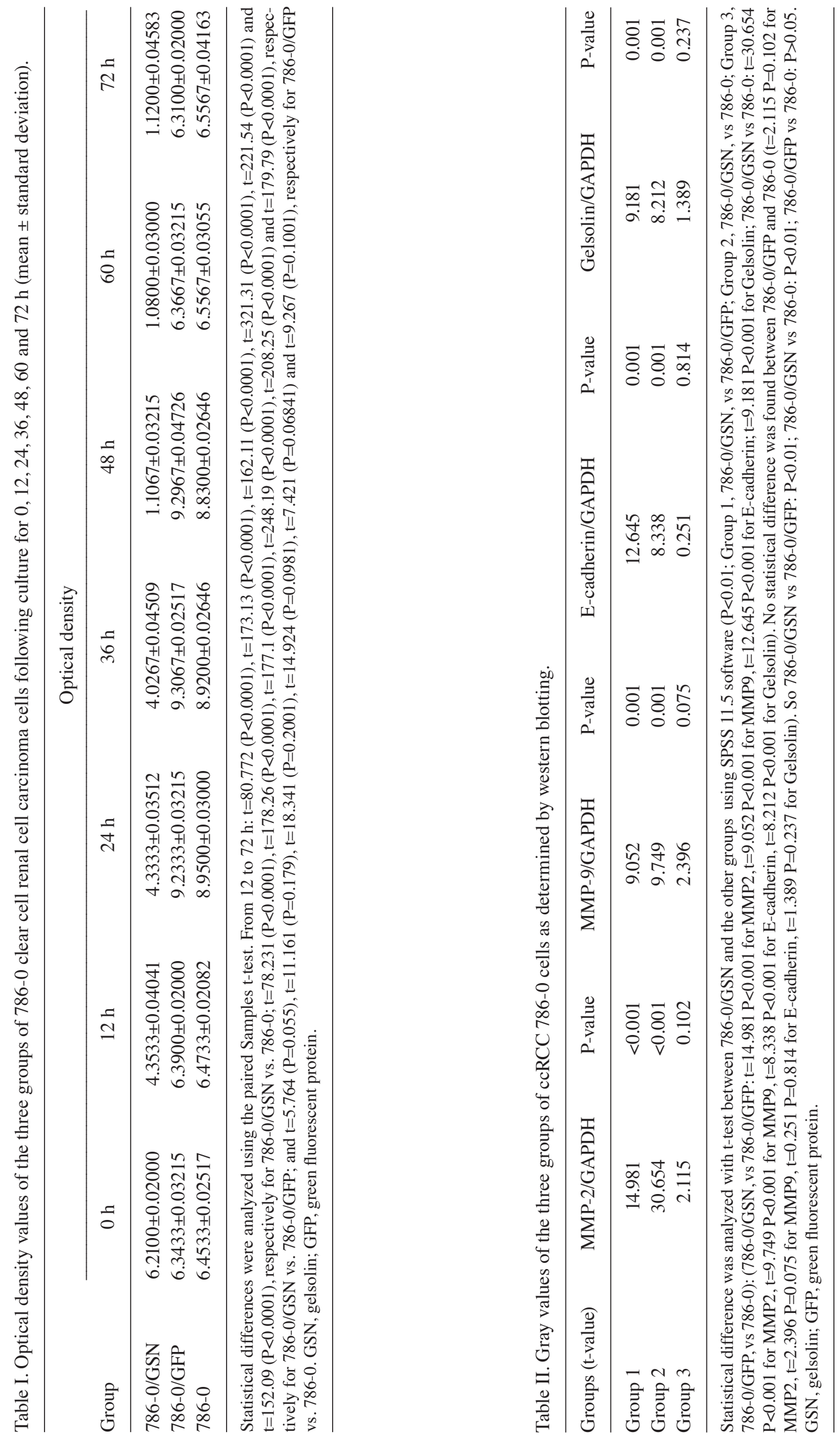
GSN (cat. no. SC-401005; Shengke Lusi Biotechnology Ltd., Shanghai, China) at $4^{\circ} \mathrm{C}$ (dilution, 1:800). The membranes were then incubated with horseradish peroxidase-conjugated antibodies (cat. no. PV6002; Zhongshan Goldenbridge Biotechnology, Beijing, China) for $30 \mathrm{~min}$ at $37^{\circ} \mathrm{C}$ (dilution, 1:800). The antigen-antibody reaction was visualized by enhanced chemiluminescence (Sigma-Aldrich) and GAPDH (cat. no. AB-82633, Abcam, Cambridge, UK) served as the internal reference.

Statistical analysis. The data were analyzed using SPSS Software version 11.5 (SPSS, Inc., Chicago, IL, USA). The data are presented as the mean \pm standard deviation and the paired Samples t-test was conducted to investigate differences within the groups for the qualitative variables. $\mathrm{P}<0.05$ was considered to indicate a statistically significant difference.

\section{Results}

Construction of the plenO-DCE-RTP-gelsolin vector and cell transfection. The plen0-DCE-RTP-gelsolin vector was constructed and identified by PCR and DNA sequencing. PCR analysis demonstrated that a single fragment was visible at $\sim 15 \mathrm{kDa}$ on $1 \%$ agarose gel (Fig. 1). In addition, DNA sequencing indicated that the recombinant plasmid contained the correct GSN gene fragment. The

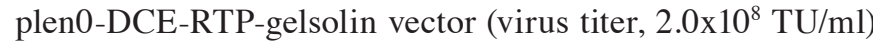
was transfected into the 786-0 ccRCC cells. Green fluorescence was apparent in the infected 786-0 cells, as observed under a fluorescence microscope, and indicated successful transfection (Fig. 2). Western blot analysis demonstrated that the expression level of GSN was markedly increased in the 786-0/GSN cells, when compared with the 786-0/GFP and 786-0 cells (Table II and Fig. 3).

Inhibition effect of GSN on the proliferation of 786-0 ccRCC cells. To investigate the effect of GSN on 786-0 cell proliferation, the cell viability of the transfected and non-transfected 786- 0 cells was measured at $0,12,24,36,48,60$ and $72 \mathrm{~h}$ using the MTT method. As shown in Fig. 4 and Table I, the OD value of the 786-0/GSN cells was significantly lower than that of the 786-0/GFP and 786-0 cells following culture for $24 \mathrm{~h}(\mathrm{P}<0.05)$, and the difference was more apparent following a longer culture period $(\mathrm{P}<0.001$ for $36-72 \mathrm{~h})$. No statistical differences were observed at the various time points between the 786-0/GFP and 786-0 groups $(\mathrm{P}>0.05)$. These results indicate that GSN may inhibit the proliferation of 786-0 cells in vitro.

Inhibition effect of GSN on the adhesion of 786-0 ccRCC cells. The effect of GSN on the adhesion ability of 786-0 cells was also examined using the MTT method. As shown in Fig. 5, the OD value of the 786-0/GSN cells (1.2600 \pm 0.02646$)$ was decreased when compared with the 786-0/GFP and 786-0 cells $(7.2533 \pm 0.04041$ and $7.3000 \pm 0.02646$, respectively). The difference between the 786-0/GSN group and the other two groups $(\mathrm{t}=214.902 ; \mathrm{P}<0.0001$ and $\mathrm{t}=279.598 ; \mathrm{P}<0.0001$ compared with the 786-0/GFP and 786-0 groups, respectively). was identified to be significant; however, no statistical difference was observed in the OD value between the 786-0/GFP

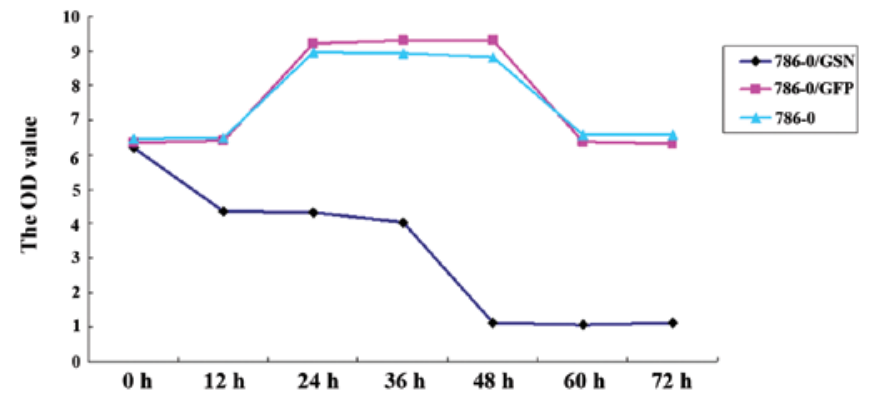

Figure 4. Comparison of the OD value between the three groups of 786-0 clear cell renal cell carcinoma cells by 3-(4,5-dimethylthiazol-2-yl)-2,5-diphenyltetrazolium bromide assay. The OD value of 786-0/GSN was lower than that of 786-0/GFP and 786-0 at 12, 24, 36, 48, 60 and $72 \mathrm{~h}(\mathrm{P}<0.05)$. OD, optical density; GSN, gelsolin; GFP, green fluorescent protein.

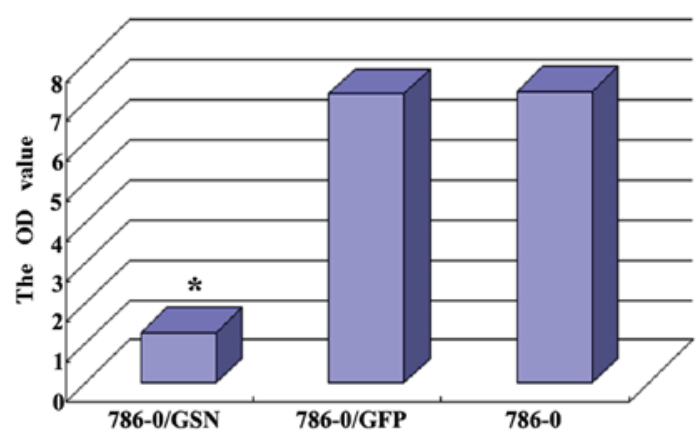

Figure 5. OD values of the three groups of 786-0 clear cell renal cell carcinoma cells as analyzed by cell adhesion assay. The OD value of the 786-0/GSN cells was significantly decreased compared with the 786-0/GFP and 786-0 cells $\left({ }^{*} \mathrm{P}<0.001\right)$. OD, optical density; GSN, gelsolin; GFP, green fluorescent protein.

group and the 786- 0 group $(\mathrm{t}=1.673 ; \mathrm{P}=0.181)$. The result revealed that the extent of $786-0 / \mathrm{GSN}$ cell adherence to the culture plates was markedly lower than that of the 786-0/GFP and 786-0 groups.

Effect of GSN on the invasion ability of 786-0 ccRCC cells. The effect of GSN on the invasion ability of 786-0 cells was observed by penetration experiments using Transwell chambers coated in Matrigel. The cells that possess invasion ability digest Matrigel and are able to penetrate the $8-\mu \mathrm{m}$ pores on the polycarbonate membrane. In the present study, fewer cells were observed to penetrate the Matrigel in the 786-0/GSN group, as compared with the cells of the 786-0/GFP and 786-0 groups (Fig. 6). The average penetration rate of the 786-0/GSN cells $(6.8000 \pm 0.83666)$ was significantly lower than that of the

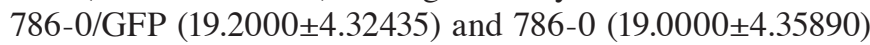
cells $(\mathrm{t}=6.295, \mathrm{P}<0.0001 ; \mathrm{t}=6.146, \mathrm{P}<0.0001)$. No statistical difference was identified in average penetration rate between the 786-0/GFP and 786-0 groups $(\mathrm{t}=0.073 ; \mathrm{P}=0.944)$.

Effect of GSN on the expression levels of MMP2, MMP9 and E-cadherin. The expression levels of MMP2 and MMP9 were detected in the transfected and non-transfected 786-0 cells using the ELISA method. As shown in Fig. 7, the expression levels of MMP2 and MMP9 were significantly decreased in the 786-0/GSN cells, and the OD value of MMP2 and MMP9 


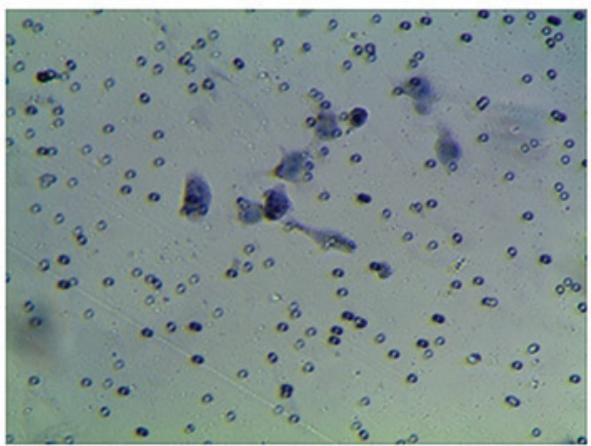

786-0/GSN

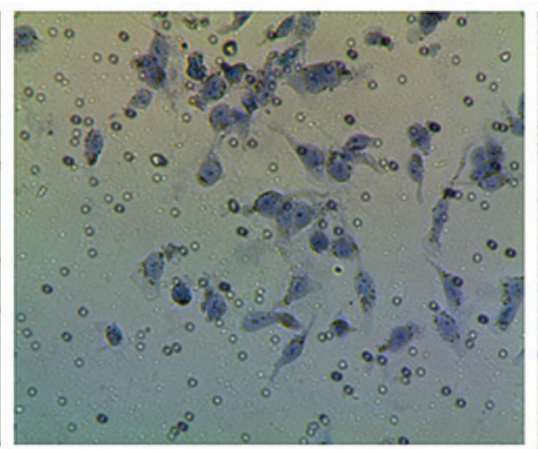

786-0/GFP

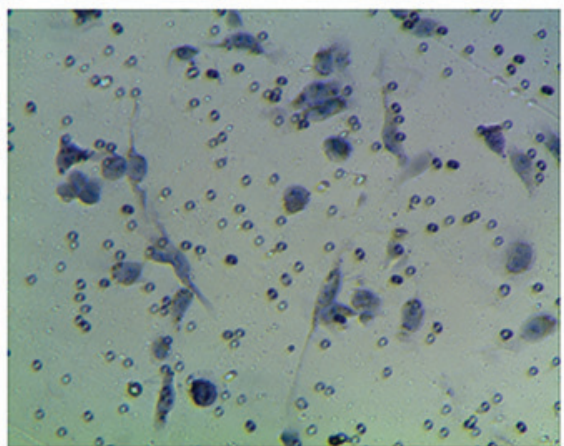

786-0

Figure 6. Transwell invasion assay displayed the penetration rate of the three groups of 786-0 clear cell renal cell carcinoma cells (magnification, $\mathrm{x} 400$ ). Fewer cells penetrated the matrigel in the 786-0/GSN group as compared with the 786-0/GFP and 786-0 group. GSN, gelsolin; GFP, green fluorescent protein.

A
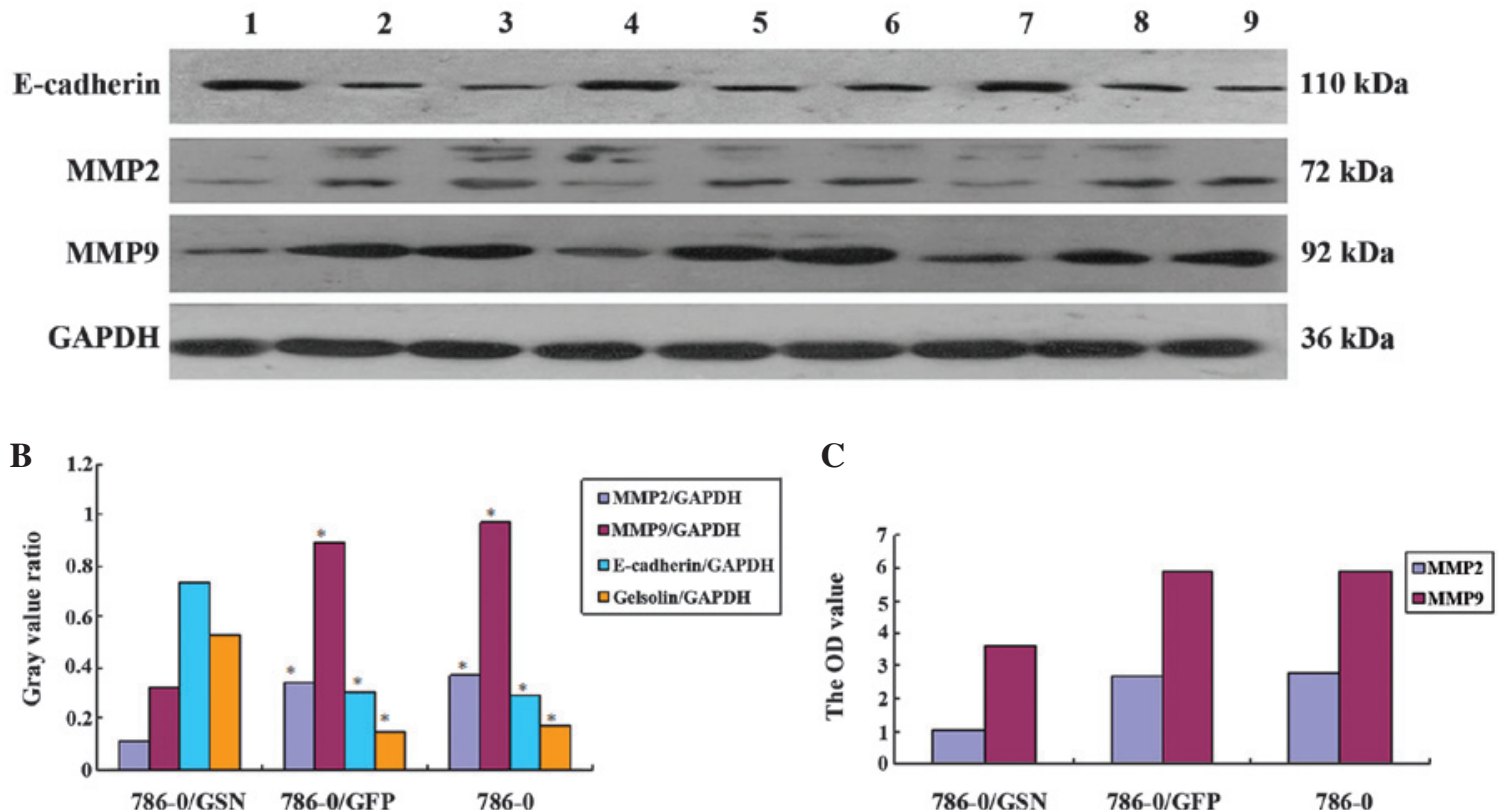

Figure 7. Effect of GSN on the level of MMP2, MMP9 and E-cadherin. (A) Western blot analysis of MMP2, MMP9 and E-cadherin expression levels. There were three specimens in each group: Lanes 1, 4 and 7, 786-0/GSN; lanes 2, 5 and 8, 786-0/GFP; lanes 3, 6 and 9, 786-0. The expression of MMP2 and MMP9 was markedly decreased in the 786-0/GSN cells, while the expression of E-cadherin was markedly increased. (B) Statistical differences were analyzed with a Samples t-test between the 786-0/GSN group and the other groups $(\mathrm{P}<0.01)$. 786-0/GSN, vs. 786-0/GFP: $\mathrm{t}=14.981$ and $\mathrm{P}<0.001$ for $\mathrm{MMP} 2$; $\mathrm{t}=9.052$ and $\mathrm{P}<0.001$ for MMP9; $\mathrm{t}=12.645$ and $\mathrm{P}<0.001$ for $\mathrm{E}$-cadherin; $\mathrm{t}=9.181$ and $\mathrm{P}<0.001$ for Gelsolin. 786-0/GSN, vs. 786-0: $\mathrm{t}=30.654$ and $\mathrm{P}<0.001$ for $\mathrm{MMP} 2 ; \mathrm{t}=9.749$ and $\mathrm{P}<0.001$ for MMP9; $\mathrm{t}=8.338$ and $\mathrm{P}<0.001$ for E-cadherin; $\mathrm{t}=8.212$ and $\mathrm{P}<0.001$ for Gelsolin. No statistical difference was found between $786-0 / \mathrm{GFP}$ and 786-0: $\mathrm{t}=2.115$ and $\mathrm{P}=0.102$ for $\mathrm{MMP} 2 ; \mathrm{t}=2.396$ and $\mathrm{P}=0.075$ for $\mathrm{MMP} 9 ; \mathrm{t}=0.251$ and $\mathrm{P}=0.814$ for $\mathrm{E}$-cadherin; $\mathrm{t}=1.389$ and $\mathrm{P}=0.237$ for $\mathrm{Gel}$ solin. $786-0 / \mathrm{GSN}$, vs. 786-0/GFP: $\mathrm{P}<0.01$; 786-0/GSN, vs. 786-0: $\mathrm{P}<0.01$; 786-0/GFP, vs. 786-0: $>0.05$. (C) The OD value of MMP2 and MMP9 in the three groups as assessed using the ELISA method. The expression level and OD value of MMP2 and MMP9 were significantly decreased in the 786-0/GSN cells as compared with the 786-0/GFP and 786-0 cells ( $<<0.0001)$. GSN, gelsolin; GFP, green fluorescent protein; MMP, matrix metalloproteinase.

in the $786-0 /$ GSN cells $(1.0400 \pm 0.03606$ and $3.6067 \pm 0.07506$, respectively) was lower than that of the 786-0/GFP (2.6633 \pm 0.08327 and $5.8633 \pm 0.03055$, respectively) and 786-0 cells $(2.7667 \pm 0.01528$ and $5.9133 \pm 0.04509$, respectively) following $48 \mathrm{~h}$ of transfection, with a significant difference between the 786-0/GSN and 786-0/GFP cells $(\mathrm{t}=30.987$, $\mathrm{P}<0.0001 ; \mathrm{t}=48.234, \mathrm{P}<0.0001)$ and between the $786-0 / \mathrm{GSN}$ and 786- 0 cells ( $\mathrm{t}=76.375, \mathrm{P}<0.0001 ; \mathrm{t}=45.629, \mathrm{P}<0.0001)$. No statistical difference was noted between the 786-0/GFP and 786- 0 cells $(\mathrm{t}=2.114, \mathrm{P}=0.161 ; \mathrm{t}=1.590, \mathrm{P}=0.197)$.

To further elucidate the effect of GSN on the expression levels of MMP2 and MMP9, the expression level of the two proteins was assessed by western blot analysis. E-cadherin was also analyzed. It was identified that in the 786-0/GSN cells, the expression levels of MMP2 and MMP9 were markedly decreased, while the expression level of E-cadherin was markedly increased (Fig. 7). Significant differences were observed between the 786-0/GSN group and the other two groups [786-0/GSN vs. 786-0/GFP: $\mathrm{t}=14.981(\mathrm{P}<0.001)$ for MMP2; $\mathrm{t}=9.052(\mathrm{P}<0.001)$ for MMP9; and $\mathrm{t}=12.645(\mathrm{P}<0.001)$ for E-cadherin; 786-0/GSN vs. 786-0: $\mathrm{t}=30.654(\mathrm{P}<0.001)$ for MMP2; $\mathrm{t}=9.749(\mathrm{P}<0.001)$ for MMP9; and $\mathrm{t}=8.338(\mathrm{P}<0.001)$ for E-cadherin]. No statistically significant difference was observed between the 786-0/GFP and 786-0 groups $[\mathrm{t}=2.115$ $(\mathrm{P}=0.102)$ for MMP2; $\mathrm{t}=2.396(\mathrm{P}=0.075)$ for MMP9; and $\mathrm{t}=0.251(\mathrm{P}=0.814)$ for E-cadherin]. 


\section{Discussion}

RCC is the most common neoplasm of the adult kidney and the most prevalent type of kidney tumor is the ccRCC subtype (4). However, the exact molecular mechanism of ccRCC remains to be elucidated. The treatment of metastatic ccRCC continues to be a challenge for clinicians and causes $\sim 35 \%$ of RCC-associated mortalities (12). The majority of RCC patients already exhibit either metastatic disease at the initial diagnosis or distant metastases following primary tumor resection (13). Since advanced ccRCC is highly resistant to chemotherapy and cytotoxic drugs, following tumor resection a third of the patients will have a recurrence. Furthermore, a quarter of the patients present locally invasive or metastatic RCC (7). This therefore led to the speculation that the prognosis of ccRCC is poor partly due to the fact that certain factors correlate with its proliferation and invasion. In recent years, particular focus has been placed on actin-binding proteins and their participation in the migratory process of cancer cells. Therefore, in the present study, GSN was selected as a novel biological marker to investigate its role in the proliferation and invasion of ccRCC.

The effect of GSN on the proliferation of 786-0 ccRCC cells was observed. An MTT assay revealed that the OD value of 786-0/GSN cells was markedly lower than that of 786-0/GFP and 786- 0 cells after being cultured for $24 \mathrm{~h}$, and the difference was more apparent with a longer culture duration. The result indicates that GSN inhibits the proliferation of 786-0 ccRCC cells. GSN is a $\mathrm{Ca}^{2+}$-activated actin-binding protein that severs F-actin filaments by breaking non-covalent bonds between the actin monomers in a polymer. It results in high-affinity complexes of GSN, which remain bound to the barbed ends of filaments, thus inhibiting extension ('capped' filaments) (14). On reduction of free intracellular $\mathrm{Ca}^{2+}$ levels, and in the presence of polyphosphoinositides, GSN is released from the barbed ends to provide sites for rapid actin filament extension. GSN is the most potent actin filament-severing protein that has been identified to date (9). The data from the present study implied that overexpression of GSN may inhibit the proliferation of 786-0 ccRCC cells. To the best of our knowledge, there are only a small number of relevant studies on the association between GSN and ccRCC, therefore, the specific pathogenesis requires further investigation.

The effect of GSN on the adhesion ability of 786-0 ccRCC cells was examined using the MTT method. The OD value of the 786-0/GSN cells was observed to be significantly decreased when compared with the 786-0/GFP and 786-0 cells. This indicated that GSN inhibits the adhesion ability of 786-0 cells. A previous study proposed that actin capping, nucleation and severing are important functions of GSN, which are required for regulation of adhesion maturation and collagen matrix remodeling. The study demonstrated that minor inhibition of the severing function of GSN, by binding to non-muscle myosin IIA, may be necessary initially to prevent actin depolymerization in the locale of adhesions (15). In addition, Ke et al (16) identified that B cell lymphoma-2 (BCL2) forms a complex with actin and GSN to decrease GSN-severing activity and increase actin polymerization, which suppresses the cell adhesion processes. The association between increased BCL2, and actin polymerization and suppression of cell adhesion was a novel observation that may provide a plausible explanation to elucidate whether BCL2 overexpression in certain tumors is correlated with improved patient survival (16). However, a fundamental characteristic of malignant and transformed cells is the aberrant organization of the actin cytoskeleton, resulting from the associated disruption of the cytoskeleton (9). This may lead to the inhibitory action of GSN overexpression on the adhesions of 786-0 cells, however, the exact mechanisms have not been clearly elucidated.

The effect of GSN on the invasion ability of 786-0 cells was evaluated using penetration experiments with Transwell chambers coated in Matrigel. Fewer cells had penetrated the Matrigel in the 786-0/GSN group, when compared with the cells of the 786-0/GFP and 786-0 groups. The average penetration rate of the 786-0/GSN cells was lower than that of the 786-0/GFP and 786-0 cells. The result indicates that overexpression of GSN may inhibit the invasion of 786-0 ccRCC cells. These findings contrast with those of existing studies. Zhuo et al (17) revealed novel functions of GSN in colorectal tumor cells, where invasion was promoted via modulation of the urokinase-type plasminogen activator cascade, with GSN potentially exerting a significant role in colorectal tumor dissemination to metastatic sites. The results of Zhuo et al (17) may be marginally attributable to the actin depolymerizing effect of GSN. However, a recent study demonstrated that the nuclear import of GSN-like actin-capping protein, as another GSN family member, and GSN had been identified to be significant in non-small cell lung cancer invasion and metastasis (18). Furthermore, De Corte et al (19) revealed that invasion induced by GSN was dependent on Ras activity, acting through the PI3K-Rac signaling pathway via the Ras guanine nucleotide exchange factor, Sos-1. These findings established a connection between GSN and the Ras oncogenic signaling pathway (19).

In order to investigate the mechanisms of GSN inhibiting the invasion of 786-0 ccRCC cells, the expression levels of MMP2, MMP9 and E-cadherin were detected in the current study. ELISA and western blot analysis revealed that GSN downregulated the expression levels of MMP2 and MMP9 in the 786-0 cells, which was consistent with previous studies $(20,21)$. Numerous molecules are involved in tumor invasion, including MMPs. MMPs are a family of related enzymes that degrade the extracellular matrix (ECM). The activation of these enzymes enables tumor cells to access the vasculature, invade target organs and develop into tumor metastases. Previous studies have indicated that full length plasma GSN is a known substrate for the MMPs, and is cleaved most efficiently by MMP3, followed by MMP2 and MMP9. Three sites that are cleaved by MMP2 and MMP9 in full length plasma GSN were identified and all were in unstructured regions (22). A recent study observed that furin cleavage of full length D187N GSN cleaved an internal $\beta$-strand in the G2 domains, which may lead to unfolding of the G2 domains that are particularly susceptible to cleavage by proteases, including the MMPs in the ECM (8). Although MMP2 and MMP9 were associated with the structure of GSN, the mechanisms of downregulating the expression levels of MMP2 and MMP9 in 786-0 ccRCC cells were not elucidated in the current study. The expression level of E-cadherin was also analyzed by western blotting in the present study; conversely, 
it was identified that GSN upregulated the expression of E-cadherin. The E-cadherin gene is a tumor suppressing gene that expresses the E-cadherin transmembrane glycoprotein, which plays a significant role in adhesion and differentiation of epithelial cells; an important protective mechanism against neoplasm formation (23). Rao et al (24) reported that alterations in the expression levels of the cytoskeletal proteins, GSN and E-cadherin, had been implicated in urothelial carcinoma tumorigenesis. Furthermore, GSN and E-cadherin possess distinctive expression patterns. GSN, but not E-cadherin, provided independent prognostic information for high-grade urothelial carcinomas. However, the mechanism underlying how the altered expression levels were associated with tumor progression was unclear. Therefore, it was hypothesized in the present study that the upregulation of E-cadherin is closely associated with GSN, so as to inhibit invasion of 786-0 ccRCC cells; although the exact pathogenesis remains unclear.

In conclusion, GSN was examined in vitro, and was observed to inhibit the proliferation and invasion of 786-0 ccRCC cells. These findings contribute to the existing knowledge on the biological functions of GSN and its effects on ccRCC. Numerous in vivo experiments on the impact of GSN in patients with ccRCC were not included in the results of the present study, which may be considered as a limitation of the study. Future investigations are required in order that more clinical samples may be collected, and the proliferation and invasion of ccRCC may be investigated in more depth. These findings may demonstrate whether GSN could serve as a novel molecular target for the development of effective therapeutic strategies to prevent the metastasis of kidney carcinoma and, therefore, improve the survival rates of patients with ccRCC.

\section{Acknowledgements}

The authors would like to thank Dr Xiaoling Zhu, Professor Limin Cai, Professor Qinggang Meng and Professor Xiaoming Jin who participated in this study, as well as their colleagues for their cooperation. The authors would also like to thank the laboratory of Wei Si Teng Company (Chongqing, China; www.cqwestern.com) for assisting with the research.

\section{References}

1. Bastola P, Stratton Y, Kellner E, Mikhaylova O, Yi Y, Sartor MA, Medvedovic M, Biesiada J, Meller J and Czyzyk-Krzeska MF: Folliculin contributes to VHL tumor suppressing activity in renal cancer through regulation of autophagy. PLoS One 8: e70030, 2013.

2. Chow WH, Dong LM and Devesa SS: Epidemiology and risk factors for kidney cancer. Nat Rev Urol 7: 245-257, 2010.

3. Ferlay J, Shin HR, Bray F, Forman D, Mathers C and Parkin DM: Estimates of worldwide burden of cancer in 2008: GLOBOCAN 2008. Int J Cancer 127: 2893-2917, 2010.

4. Kovacs G, Akhtar M, Beckwith BJ, Bugert P, Cooper CS, Delahunt B, Eble JN, Fleming S, Ljungberg B, Medeiros LJ, et al: The Heidelberg classification of renal cell tumours. J Pathol 183: 131-133, 1997.

5. Ramakrishnan S, Ellis L and Pili R: Histone modifications: Implications in renal cell carcinoma. Epigenomics 5: 453-462, 2013.
6. Kim HL, Seligson D, Liu X, Janzen N, Bui MH, Yu H, Shi T, Figlin RA, Horvath $S$ and Belldegrun AS: Using protein expressions to predict survival in clear cell renal carcinoma. Clin Cancer Res 10: 5464-5471, 2004

7. Chuang MJ, Sun KH, Tang SJ, Deng MW, Wu YH, Sung JS, Cha TL and Sun GH: Tumor-derived tumor necrosis factor-alpha promotes progression and epithelial-mesenchymal transition in renal cell carcinoma cells. Cancer Sci 99: 905-913, 2008.

8. Solomon JP, Page LJ, Balch WE and Kelly JW: Gelsolin amyloidosis: Genetics biochemistry pathology and possible strategies for therapeutic intervention. Crit Rev Biochem Mol Biol 47: 282-296, 2012.

9. Li GH, Arora PD, Chen Y, McCulloch CA and Liu P: Multifunctional roles of gelsolin in health and diseases. Med Res Rev 32: 999-1025, 2012.

10. Baig RM, Mahjabeen I, Sabir M, Masood N, Ali K, Malik FA and Kayani MA: Mutational spectrum of gelsolin and its down regulation is associated with breast cancer. Dis Markers 34: 71-80, 2013.

11. Visapää H, Bui M, Huang Y, Seligson D, Tsai H, Pantuck A, Figlin R, Rao JY, Belldegrun A, Horvath S and Palotie A: Correlation of $\mathrm{Ki}-67$ and gelsolin expression to clinical outcome in renal clear cell carcinoma. Urology 61: 845-850, 2003.

12. Siegel R, Naishadham D and Jemal A: Cancer statistics 2012. CA Cancer J Clin 62: 10-29, 2012.

13. Finley DS, Pantuck AJ and Belldegrun AS: Tumor biology and prognostic factors in renal cell carcinoma. Oncologist 16 (Suppl 2): S4-S13, 2011.

14. Nag S, Larsson M, Robinson RC and Burtnick LD: Gelsolin: The tail of a molecular gymnast. Cytoskeleton (Hoboken) 70: 360-384, 2013.

15. Arora PD, Wang Y, Bresnick A, Dawson J, Janmey PA and $\mathrm{McCulloch} \mathrm{CA}$ : Collagen remodeling by phagocytosis is determined by collagen substrate topology and calcium-dependent interactions of gelsolin with nonmuscle myosin IIA in cell adhesions. Mol Biol Cell 24: 734-747, 2013.

16. Ke H, Zhang JY, Akiyama SK and French JE: BCL2 interaction with actin in vitro may inhibit cell motility by enhancing actin polymerization. Cell Adh Migr 5: 6-10, 2011.

17. Zhuo J, Tan EH, Yan B, Tochhawng L, Jayapal M, Koh S, Tay HK, Maciver SK, Hooi SC, Salto-Tellez M, et al: Gelsolin induces colorectal tumor cell invasion via modulation of the urokinase-type plasminogen activator cascade. PloS One 7: e43594, 2012.

18. Zhu WY, Hunag YY, Liu XG, He JY, Chen DD, Zeng F, Zhou JH and Zhang YK: Prognostic evaluation of CapG gelsolin P-gp GSTP1 and Topo-II proteins in non-small cell lung cancer. Anat Rec (Hoboken) 295: 208-214, 2012.

19. De Corte V, Bruyneel E, Boucherie C, Mareel M, Vandekerckhove J and Gettemans J: Gelsolin-induced epithelial cell invasion is dependent on ras-rac signaling. EMBO J 21: 6781-6790, 2002.

20. Liao CJ, Wu TI, Huang YH, Chang TC, Wang CS, Tsai MM, Hsu CY, Tsai MH, Lai CH and Lin KH: Overexpression of gelsolin in human cervical carcinoma and its clinicopathological significance. Gynecol Oncol 120: 135-144, 2011.

21. Zhan L, Zhang H, Zhang Q,Woods CG, Chen Y, Xue P, Dong J, Tokar EJ, Xu Y, Hou Y, et al: Regulatory role of KEAP1 and NRF2 in PPAR $\gamma$ expression and chemoresistance in human non-small-cell lung carcinoma cells. Free Radic Biol Med 53: 758-768, 2012.

22. Park SM, Hwang IK, Kim SY, Lee SJ, Park KS and Lee ST: Characterization of plasma gelsolin as a substrate for matrix metalloproteinases. Proteomics 6: 1192-1199, 2006.

23. Anbiaee R, Mojir Sheibani K, Torbati P and Jaam H: Abnormal expression of e-cadherin in gastric adenocarcinoma and its correlation with tumor histopathology and helicobacter pylori infection. Iran Red Crescent Med J 15: 218-222, 2013.

24. Rao J, Seligson D, Visapaa H, Horvath S, Eeva M, Michel K, Pantuck A, Belldegrun A and Palotie A: Tissue microarray analysis of cytoskeletal actin-associated biomarkers gelsolin and E-cadherin in urothelial carcinoma. Cancer 95: 1247-1257, 2002. 